\title{
SKEW-PRODUCTS WITH SIMPLE APPROXIMATIONS
}

\author{
P. N. WHITMAN
}

\begin{abstract}
Conditions are given in order that the cartesian product of two measure-preserving invertible transformations admits an approximation. A class of skew-product transformations is defined and conditions are given for a member of this class to admit a simple approximation.
\end{abstract}

1. Preliminaries. Let $(X, \mathcal{F}, \mu)$ be a Lebesgue space; that is, a measure space isomorphic to the unit interval with Lebesgue measure. A measurepreserving invertible point transformation of $X$ is called an automorphism of $(X, \mathscr{F}, \mu)$.

Let $T: X \rightarrow X$ be an automorphism. The induced automorphism $T_{A}$ : $A \rightarrow A$ where $A \subset \mathcal{F}$ is defined as follows:

$$
T_{A} x=T^{k} x, \quad x \in A
$$

where $k$ is the least positive integer such that $T^{k} x \in A$.

Let $Z$ denote the set of positive integers. Let $f: X \rightarrow Z$ be an integrable function. The special automorphism over $T$ built under the function $f$ is defined as follows:

$$
\begin{aligned}
& \text { Put } B(k, n)=\{(x, n): x \in X, f(x)=k\}, n, k \in Z \text { and } 1 \leqslant \\
& n \leqslant k \text {. Put } X(f)=\cup_{k \geqslant 1} \cup_{1 \leqslant n \leqslant k} B(k, n) \text {. Identify } X \text { with } \\
& \text { the set } \cup_{k \geqslant 1} B(k, 1) .
\end{aligned}
$$

We may regard each set $B(k, n), 1 \leqslant n \leqslant k$, as a copy of $B(k, 1)$. Consequently we may extend the measure $\mu$ to $X(f)$ and form a normalised measure $\mu^{\prime}$ on $X(f)$ in the obvious way. We define $T_{f}$, the special automorphism over $T$, by

$$
\begin{gathered}
T_{f}(x, n)=(x, n+1) \quad \text { if } 1 \leqslant n<f(x), \\
T_{f}(x, f(x))=(T x, 1) .
\end{gathered}
$$

The following definitions are due to Katok and Stepin [4] and Chacon [1] respectively.

DeFINITION 1. An automorphism $T$ is said to admit a cyclic approximation by periodic transformations of the first kind (a.p.t.I) with speed $f(n)$, where $f(n)$ is a sequence of real numbers decreasing to zero, if there exists a sequence of partitions $\{\xi(n)\}, \xi(n)=\left\{C_{i}(n): 1 \leqslant i \leqslant q(n)\right\}$ such that:

1. $\xi(n) \rightarrow \varepsilon_{X}$

2. $\sum_{i=1}^{q(n)} \mu\left(T C_{i}(n) \Delta C_{i+1}(n)\right)<f(q(n))$, where $C_{q(n)+1}(n)$ means $C_{1}(n)$.

Received by the editors February 8, 1978.

AMS (MOS) subject classifications (1970). Primary 28A65. 
Definition 2. An automorphism $T$ admits a simple approximation if there exists a sequence of partitions $\{\xi(n)\}, \xi(n)=\left\{C_{i}(n): 1 \leqslant i \leqslant q(n)\right\}$ such that:

1. $\xi(n) \rightarrow \varepsilon_{X}$

2. $T C_{i}(n)=C_{i+1}(n), 1 \leqslant i \leqslant q(n)-1$.

Let $R$ denote the positive reals.

We also require a slight adaption of Definition 1 , as follows.

Definition 3. An automorphism $T$ admits a cyclic a.p.t.I with speed $f(x)$, if $T$ admits a cyclic a.p.t.I with speed $f(n)$, where $f: R \rightarrow R$ and $f(x) \rightarrow 0$ as $x \rightarrow \infty$.

In [6] the following two results were shown.

TheOREM 1. Let $T: X \rightarrow X$ admit a cyclic a.p.t.I with speed $f(n)=o\left(1 / n^{2}\right)$ with respect to a sequence of partitions $\xi(n), \xi(n)$ having $q(n)$ elements. Let $A \in \mathcal{F}$ be approximated by sets $A(n) \leqslant \xi(n)$ with $A(n) \subset A$ such that $\mu(A \backslash A(n))=o(1 / q(n))$. Then $T_{A}$ admits a simple approximation.

THEOREM 2. Let $T: X \rightarrow X$ admit a cyclic a.p.t.I with speed $g(x)=o\left(1 / x^{k}\right)$ with respect to a sequence of partitions $\{\xi(n)\}, \xi(n)=\left\{C_{i}(n): 1 \leqslant i \leqslant q(n)\right\}$. Let $f: X \rightarrow Z$ be integrable with $f\left(C_{i}(n)\right)=k_{i}(n) \in Z, 1 \leqslant i \leqslant q(n), n \leqslant 1$. Then $T_{f}$ admits a cyclic a.p.t.I with speed $G(n)=o\left(1 / n^{k}\right)$.

2. Approximation of products. Let $(X, \mathscr{F}, \mu)$ and $(Y, \mathscr{D}, \nu)$ be Lebesgue spaces. Let $S$ and $T$ be automorphisms of $X$ and $Y$ respectively. Let $\xi(n)=\left\{C_{i}(n): 1 \leqslant i \leqslant p(n)\right\}$ and $\zeta(n)=\left\{D_{j}(n): 1 \leqslant j \leqslant q(n)\right\}$ be partitions in $X$ and $Y$ respectively. Define $S_{n}$ and $T_{n}$ by

$$
S_{n} C_{i}(n)=C_{i+1}(n), \quad 1 \leqslant i \leqslant p(n) \text {, where } C_{p(n)+1}(n)
$$

means $C_{1}(n)$, and

$$
T_{n} D_{j}(n)=D_{j+1}(n), \quad 1 \leqslant j \leqslant q(n), \text { where } D_{q(n)+1}(n)
$$

means $D_{1}(n)$.

The lemma below is easily verified.

LEMMA 1. If $(p(n), q(n))=1$, then $S_{n} \times T_{n}$ maps the elements of $\xi(n) \times$ $\zeta(n)$ cyclically; that is

$$
S_{n} \times T_{n}\left(C_{i}(n) \times D_{j}(n)\right)=C_{i+1}(n) \times D_{j+1}(n)
$$

and $\left(S_{n} \times T_{n}\right)^{k}\left(C_{i}(n) \times D_{j}(n)\right) \neq C_{i}(n) \times D_{j}(n)$ for $k<p(n) q(n)$.

The theorem which follows gives the conditions for a product of automorphisms to admit a cyclic a.p.t.I with speed of the form $O\left(1 / n^{k}\right)$.

THEOREM 3. Let $S: X \rightarrow X$ admit a cyclic a.p.t.I with speed $f(n)$ $O\left(1 / n^{(r+1) k}\right)$ where $r \geqslant 2, k \geqslant 1, r, k \in Z$, with respect to a sequence of partitions $\{\xi(n)\}, \xi(n)=\left\{C_{i}(n): 1 \leqslant i \leqslant p(n)\right\}$. Let $T: Y \rightarrow Y$ admit a cyclic a.p.t.I with speed $g(n)=O\left(1 / n^{(r+1) k}\right)$, with respect to a sequence of partitions $\{\zeta(n)\}, \zeta(n)=\left\{D_{j}(n): 1 \leqslant j \leqslant q(n)\right\}$. Suppose that: 
1. $(p(n), q(n))=1$;

2. $p(n)<q(n)$;

3. $p(n)^{r}>q(n)$.

Then $S \times T$ admits a cyclic a.p.t.I with speed $G(n)=2 \delta / n^{k}$, for some $\delta>0$.

Proof.

$$
\begin{aligned}
& \sum_{i=1}^{p(n)} \sum_{j=1}^{q(n)} \mu \times \nu\left(S \times T\left(C_{i}(n) \times D_{j}(n)\right) \Delta\left(C_{i+1}(n) \times D_{j+1}(n)\right)\right) \\
& \quad \leqslant \sum_{i} \sum_{j} \mu\left(C_{i}(n)\right) \nu\left(T D_{j}(n) \Delta D_{j+1}(n)\right)+\nu\left(D_{j}(n)\right) \mu\left(S C_{i}(n) \Delta C_{i+1}(n)\right) \\
& \leqslant p(n) \mu\left(C_{1}(n)\right) \sum_{j} \nu\left(T D_{j}(n) \Delta D_{j+1}(n)\right) \\
& \quad+q(n) \nu\left(D_{1}(n)\right) \sum_{i} \mu\left(S C_{i}(n) \Delta C_{i+1}(n)\right) \\
& \quad \leqslant \sum_{j} \nu\left(T D_{j}(n) \Delta D_{j+1}(n)\right)+\sum_{i} \mu\left(S C_{i}(n) \Delta C_{i+1}(n)\right) \\
& <f(p(n))+g(q(n)) .
\end{aligned}
$$

Since $f(n)=O\left(1 / n^{(r+1) k}\right), f(n)<\delta_{1} / n^{(r+1) k}$ for some $\delta_{1}>0$. Similarly, $g(n)<\delta_{2} / n^{(r+1) k}$ for some $\delta_{2}>0$. Put $\delta=\max \left\{\delta_{1}, \delta_{2}\right\}$ then

$$
f(p(n))+g(q(n))<\delta / p(n)^{(r+1) k}+\delta / q(n)^{(r+1) k}<2 \delta / p(n)^{k} q(n)^{k}
$$

since by (3) $p(n)^{r}>q(n)$.

Put $G(n)=2 \delta / n^{k}$. By Lemma 1 and the above, $\{\xi(n) \times \zeta(n)\}$ is a sequence of partitions with respect to which $S \times T$ admits a cyclic a.p.t.I with speed $g(n)$.

In [1] it is shown that if an automorphism admits a cyclic a.p.t.I with speed $\theta / n, \theta<1$, then it has simple spectrum. Consequently if $\delta<\frac{1}{2}$ and $k \geqslant 1$, then $S \times T$ will have simple spectrum. If $f(n)=o\left(1 / n^{(r+1) k}\right)$ and $g(n)=$ $o\left(1 / n^{(r+1) k}\right)$ then it is easily seen that $S \times T$ will have speed of approximation $G(n)=2 \delta / n^{k}$ for any $\delta>0$.

If $S$ and $T$ and $S \times T$ all have simple spectrum then $S$ and $T$ can have no common spectral type and consequently by a result of Hahn and Parry [3] $S$ and $T$ are disjoint.

If $T$ has simple spectrum then $T \times T$ has spectral multiplicity strictly greater than one. Consequently Theorem 3 shows that there are restrictions on the types of approximating partitions which exist for $T$, when the speed of approximation is of the order of $O\left(1 / n^{3}\right)$.

In a similar way to Theorem 3 we can also show the following.

THEOREM 4. Let $S: X \rightarrow X$ admit a cyclic a.p.t.I with speed $f(n)=a / \log n$, $a>0$, with respect to a sequence of partitions $\{\xi(n)\}, \xi(n)$ having $p(n)$ elements. Let $T: Y \rightarrow Y$ admit a cyclic a.p.t.I with speed $g(n)=b / \log n, b>0$, with 
respect to a sequence of partitions $\{\zeta(n)\}, \zeta(n)$ having $q(n)$ elements. Suppose $p(n)$ and $q(n)$ satisfy:

1. $(p(n), q(n))=1$;

2. $p(n)<q(n)$;

3. $\log q(n)<k \log p(n)$.

Then $S \times T$ admits a cyclic a.p.t.I with speed $g(n)=(k+1)(a+b) / \log n$.

3. Skew-products with simple approximations. The class of skew-products we shall consider were discussed by Newton in [5] where formulae were given for calculating their entropy. Goodson has considered skew-products of a different type in [2]. He has given conditions for finite skew-products to admit a simple approximation.

Let $S$ and $T$ be automorphisms of $X$ and $Y$ respectively. Let $f: X \rightarrow Z$ be integrable. The skew-products considered below are of the form

$$
\psi(x, y)=\left(S x, T^{f(x)} y\right), \quad x \in X, y \in Y .
$$

Let $\eta$ be the measure which assigns measure 1 to each point of $Z$. Let $V$ be the subset of $X \times Y \times Z$ defined by $(x, y, i) \in V$ if $i \leqslant f(x)$. So that $V=V^{\prime} \times Y$ where $V^{\prime}$ is the subset of $X \times Z$ defined by $(x, i) \in V^{\prime}$ if $i \leqslant f(x)$. It is easily seen that

$$
\mu \times \nu \times \eta(V)=\mu \times \eta\left(V^{\prime}\right)=\int f(x) d \mu .
$$

We can consider $V$ as a Lebesgue space with normalised measure $\mu^{\prime}$ defined by

$$
\mu^{\prime}(A)=\mu \times \nu \times \eta(A) \cdot\left(\int f(x) d \mu\right)^{-1}
$$

where $A \subset V$.

Define an automorphism $\phi$ on $V$ by

$$
\begin{aligned}
\phi(x, y, i) & =(x, T y, i+1) & \text { if } i<f(x), \\
& =(S x, T y, 1) & \text { if } i=f(x) .
\end{aligned}
$$

Then $\phi=S_{f} \times T$. Furthermore it is clear that $\psi$ is the automorphism induced by $\phi$ on the set $X \times Y \times\{1\}$.

Definition 4. Let $\xi$ be a partition in $X$ such that every element of $\xi$ is contained in exactly one of the sets $B(k, 1)$, defined in the first section, for some $k$. Order the sets $B(k, n), k \geqslant 1,1 \leqslant n \leqslant k$, lexicographically. Then $\xi^{f}$ is the partition in $X(f)$ consisting of the elements $C \in \xi$, together with for each $C \in \xi$, where $C \subset B(k, 1)$, a copy of $C$ in each of the sets $B(k, n)$, $1 \leqslant n \leqslant k$. The ordering on $\xi^{f}$ is that inherited from the sets $B(k, n)$.

We now give conditions in order that $\psi$ should admit a simple approximation.

THEOREM 5. Let $S: X \rightarrow X$ admit a cyclic a.p.t.I with speed $h(x)=$ $O\left(1 / x^{3(r+1)}\right), r \geqslant 2, r \in Z$, with respect to a sequence of partitions $\{\xi(n)\}$, 
$\xi(n)=\left\{C_{i}(n): 1 \leqslant i \leqslant s(n)\right\}$. Let $f: X \rightarrow Z$ be integrable with $f\left(C_{i}(n)\right)=$ $k_{i}(n), 1<i \leqslant s(n), n \geqslant 1$, and suppose that $\xi^{f}(n)$ has $p(n)$ elements. Let $T$ : $Y \rightarrow Y$ admit a cyclic a.p.t.I with speed $g(n)=O\left(1 / n^{3(r+1)}\right)$ with respect to a sequence of partitions $\{\zeta(n)\}, \zeta(n)=\left\{D_{j}(n): 1 \leqslant j \leqslant q(n)\right\}$. Suppose that:

1. $(p(n), q(n))=1$

2. $p(n)<q(n)$

3. $p(n)^{r}>q(n)$;

4. $s(n)^{r+1} \mu\left(X \backslash \cup_{i=1}^{s(n)} C_{i}(n)\right) \rightarrow 0$ as $n \rightarrow \infty$;

5. $q(n)^{2} \nu\left(Y \backslash \cup \cup j=1\right.$ 界 $\left.D_{j}(n)\right) \rightarrow 0$ as $n \rightarrow \infty$.

Then $\psi(x, y)=\left(S x, T^{f(x)} y\right)$ admits a simple approximation.

Proof. By Theorem 2, $S_{f}$ admits a cyclic a.p.t.I with speed $H(n)=$ $O\left(1 / n^{3(r+1)}\right)$, with respect to the sequence of partitions $\xi^{f}(n)$.

By the remarks following Theorem $3, \phi$ admits a cyclic a.p.t.I with speed $G(n)=O\left(1 / n^{2}\right)$ with respect to the sequence of partitions $\left\{\xi^{f}(n) \times \zeta(n)\right\}$. Now

$$
\begin{aligned}
& p(n) q(n) \mu^{\prime}\left(X \times Y \times\{1\} \backslash \sum_{i=1}^{s(n)} \sum_{j=1}^{q(n)} C_{i}(n) \times D_{j}(n) \times\{1\}\right) \\
& \leqslant p(n) q(n) \mu\left(X \backslash \sum_{i=1}^{s(n)} C_{i}(n)\right)+p(n) q(n) \nu\left(Y \backslash \sum_{j=1}^{q(n)} D_{j}(n)\right) \\
& \leqslant p(n)^{r+1} \mu\left(X \backslash \bigcup_{i} C_{i}(n)\right) q\left(n^{2}\right) \nu\left(Y \backslash \bigcup_{j} D_{j}(n)\right) \rightarrow 0 \text { as } n \rightarrow \infty,
\end{aligned}
$$

since $p(n) \leqslant s(n)$. $\left(1+2 \int f d \mu\right)$ for $n$ sufficiently large.

Hence $\phi_{X \times Y \times\{1\}}$ admits a simple approximation by Theorem 1, which completes the proof.

It is fairly easy to manufacture examples of automorphisms $S$ and $T$ which satisfy the conditions of Theorem 5 by the stacking method. Using methods similar to those in [4], we can use continued-fraction theory to provide rotations of the unit interval, $S$ and $T$, which satisfy the conditions of Theorem 3. As a consequence of this, it is possible to give examples of skew-products, of the type discussed above, with interval exchange transformations in the base, and rotations in the fibres, which have simple spectrum, without using Theorem 5 .

Chacon [1] has generalised the idea of cyclic a.p.t.I to that of approximation with multiplicity $N$. We remark that all the results shown above have straightforward generalisations to the 'multiplicity $N$ situation'. We then have the following generalisation of Theorem 5 .

THEOREM 6. Let $S, f$ and $T$ be as in Theorem 5. Suppose that:

1. g.c.d. $(p(n), q(n))=N$;

2. $p(n)<q(n)$ 
3. $p(n)^{r}>q(n)$

4. $s(n)^{r+1} \mu\left(X \backslash \cup_{i=1}^{s(n)} C_{i}(n)\right) \rightarrow 0$ as $n \rightarrow \infty$;

5. $q(n)^{2} \nu\left(Y \backslash \cup_{j=1}^{q(n)} D_{j}(n)\right) \rightarrow 0$ as $n \rightarrow \infty$.

Then $\psi(x, y)=\left(S x, T^{f(x)} y\right)$ admits a simple approximation with multiplicity $N$.

\section{BIBLIOGRAPHY}

1. R. V. Chacon, Approximations and spectral multiplicity, Lecture Notes in Math., vol. 160, Springer-Verlag, Berlin and New York, 1970, pp. 18-27. MR 42 \#6186.

2. G. R. Goodson, Skew-products with simple spectrum, J. London Math. Soc. (2) 10 (1975), 441-446. MR 52 \#703.

3. F. Hahn and W. Parry, Some characteristic properties of dynamical systems with quasi-discrete spectrum, Math Systems Theory 2 (1968), 179-190. MR 37 \#6435.

4. A. B. Katok and A. M. Stepin, Approximations in ergodic theory, Russian Math. Surveys (5) 22 (1967), 77-102. MR 36 \#2776.

5. D. Newton, On the entropy of certain classes of skew-product transformations, Proc. Amer. Math. Soc. 21 (1969), 722-726. MR 40 \# 1581.

6. P. N. Whitman, Approximation of induced automorphisms and special automorphisms, Proc. Amer. Math. Soc. 70 (1978), 139-145.

University of the Witwatersrand, Jan Smuts AVenue, JohanNesburg, South Africa 